\title{
Chronic Treatment with Desipramine Improves Cognitive Performance of Rats in an Attentional Set-Shifting Test
}

\author{
M Danet S Lapiz', Corina O Bondi' and David A Morilak*,' \\ 'Department of Pharmacology and Center for Biomedical Neuroscience, University of Texas Health Science Center, San Antonio, TX, USA
}

\begin{abstract}
Alterations in central monoaminergic neurotransmission are important in the actions of many antidepressants. This study tested the hypothesis that tonic elevation of noradrenergic (NA) neurotransmission in medial prefrontal cortex (mPFC) by chronic treatment with the selective norepinephrine (NE) reuptake blocker desipramine (DMI) may contribute to the beneficial cognitive effects of this antidepressant drug (AD). Male Sprague-Dawley rats were treated with DMl acutely ( $15 \mathrm{mg} / \mathrm{kg}$, i.p.) or chronically for 21 days (7.5 mg/ $\mathrm{kg} /$ day via osmotic minipump) before assessing performance on an attentional set-shifting test. The extradimensional set-shifting component of this test reflects a process of cognitive flexibility that is dependent upon mPFC, and that we have shown previously to be facilitated by NA activity in mPFC. Microdialysis was performed to measure NE release in mPFC concurrently with behavioral testing. Acute DMI treatment produced an increase in extracellular NE levels in $\mathrm{mPFC}$, and a modest improvement in overall performance across all task stages of the attentional set-shifting test, but failed to produce a significant improvement in any of the individual specific tasks comprising the test sequence. Chronic DMI treatment tonically elevated basal extracellular NE levels in mPFC, associated with a significant improvement in performance specifically on the extradimensional set-shifting component of the test. There was also a significant reduction in set loss errors in rats treated chronically with DMI. Hence, tonic elevation of NA transmission in mPFC by chronic DMI treatment was associated with a time-dependent facilitation of cognitive flexibility that may contribute to the mechanism whereby chronic treatment with ADs, specifically NE reuptake blockers, may exert a beneficial therapeutic effect on cognition in depressed patients.
\end{abstract}

Neuropsychopharmacology (2007) 32, I000-1010. doi: I0.1038/sj.npp. I 30 I235; published online I November 2006

Keywords: arousal; attention; depression; desipramine; medial prefrontal cortex; norepinephrine

\section{INTRODUCTION}

Alterations in brain noradrenergic (NA) neurotransmission are important in the actions of many antidepressant drugs (ADs), particularly the older class of tricyclic ADs and the newer class of dual uptake inhibitors (Delgado and Moreno, 2000; Frazer, 2000, 2001; Gorman and Sullivan, 2000; Morilak and Frazer, 2004). Moreover, in addition to the older norepinephrine (NE)-specific tricyclic drug, desipramine (DMI), a newer generation of selective NE reuptake inhibitors have emerged recently as effective treatments for affective disorders (reviewed in Frazer, 2000, 2001; Morilak and Frazer, 2004). Activation of the brain NA system exerts a widespread modulatory influence that facilitates a variety of autonomic, neuroendocrine, cognitive, and behavioral

*Correspondence: Dr DA Morilak, Department of Pharmacology, MC 7764, University of Texas Health Science Center, 7703 Floyd Curl Drive, San Antonio, TX 78229-3900, USA, Tel: + I 210567 4174, Fax: + I 210567 4303, E-mail: morilak@uthscsa.edu

Received I August 2006; revised I September 2006; accepted 15 September 2006

Online publication: 19 September 2006 at http://www.acnp.org/ citations/Npp0919060605 I 7/default.pdf processes, which in turn may contribute to behavioral state changes such as increased arousal, or to adaptive responses to acute stress (Aston-Jones et al, 1991, 1999, 2000; Morilak et al, 2005). In this way, enhancing NA function may be beneficial in the treatment of depression and anxiety (see Morilak and Frazer, 2004).

It is increasingly recognized that in addition to mood symptoms, cognitive dysfunction is also a major component of depression and anxiety disorders, and may in fact underlie many of the affective symptoms (Anisman and Matheson, 2005; Beck, 1976; Coles and Heimberg, 2002; Mathews and Mackintosh, 1998). Cognitive and emotional biases have long been considered important in the development and maintenance of depression, particularly in response to stress (Beck, 1976). Neuropsychological studies of depressed patients have revealed impairments in executive functions related to frontal lobe activity, for example, verbal fluency, cognitive set-shifting, behavioral flexibility, and perseveration (see Fossati et al, 1999). Depressed patients show abnormal responses to performance feedback, reduced motivation, diminished cognitive capacity, a narrowing of attentional focus to depressionrelevant thoughts, and difficulty in shifting attentional set 
from one affective dimension to another (Murphy et al, 1999). This is consistent with the perseverative attention to themes of loss and worthlessness and the persistent ruminations that are prevalent in this disorder (Murphy et al, 1999, 2003). Imaging studies have shown associations between cognitive dysfunction and altered activity in prefrontal cortex in depression (Rogers et al, 2004; Sheline, 2003). Treatment with cognitive behavioral therapy or AD drugs such as paroxetine produced changes in prefrontal cortical function, also revealed by functional neuroimaging (Goldapple et al, 2004; Kennedy et al, 2001; Prasko et al, 2004).

In primates, the medial prefrontal cortex (mPFC) mediates the ability to shift attention between perceptual features of complex stimuli (Dias et al, 1996, 1997; Owen et al, 1991), and in rats, it has been implicated specifically in attentional set-shifting, or the ability to 'unlearn' an established contingency in order to learn a new one by shifting attention from a previously salient stimulus dimension to a previously irrelevant one (Birrell and Brown, 2000). Lesions of rat mPFC resulted in a state of 'emotional perseveration', or a failure to unlearn old contingencies in a fear-conditioning extinction paradigm (Morgan et al, 2003).

Thus, mPFC is implicated in the ability to attend to changes in the environment and adapt behavioral response strategies accordingly, that is, behavioral flexibility (Dalley et al, 2004; Ragozzino et al, 1999), and several tests have been developed to measure this cognitive ability in both humans and animals (Birrell and Brown, 2000; Dias et al, 1996). One such test is the Wisconsin Card Sorting Test (WCST), used to assess strategy-switching deficits in patients with frontal lobe damage or dysfunction (see Tollefson, 1996). To address more specifically the different components of behavioral flexibility assessed in the WCST, Roberts et al (1992) developed a visual discrimination task to investigate attentional set formation in both humans and primates. The test required a series of discriminations using stimuli that varied along two visual dimensions (eg, colored shapes and white lines). Each new discrimination required the subjects to attend to either the same dimension, or to switch their attention to the previously distracting dimension (an extradimensional shift, ED) (Roberts et al, 1992). Birrell and Brown (2000) subsequently developed a similar test to assess attentional set-shifting performance in rats. In this test, rats are trained to dig for a food reward in small pots containing distinctive digging media and marked with different odors. The rats must learn which of these stimulus dimensions (odor or digging medium) is salient in signaling the food reward. After learning a given contingency, the rules are changed as in the WCST, and the rats must unlearn the prior contingency in order to learn a new one. In this way, the rats are tested on a series of increasingly difficult tasks including stimulus reversals, intra- and extradimensional (ED) set shifts. Using this test, lesions of rat mPFC were shown to interfere specifically with ED setshifting capability, reflecting an inability to attend to a previously irrelevant stimulus dimension and ignore the previously salient dimension that is no longer informative (Birrell and Brown, 2000). Likewise, selective deafferentation of NE terminals in rat $\mathrm{MPFC}$ also impaired ED setshifting (Eichenbaum et al, 2003), and more recently, we have shown that elevating NA activity at $\alpha_{1}$ adrenergic receptors in mPFC facilitated performance on this task (Lapiz and Morilak, 2006). Thus, in the present study, we tested the hypothesis that tonic elevation of NA transmission in $\mathrm{mPFC}$ produced by chronic treatment with the selective NE reuptake blocker desipramine (DMI) may contribute to the beneficial cognitive effects of this $\mathrm{AD}$. Portions of this work have been presented in abstract form (Lapiz and Morilak, 2005; Petre et al, 2005).

\section{MATERIALS AND METHODS}

\section{Animals}

A total of 50 adult male Sprague-Dawley rats, weighing 200$225 \mathrm{~g}$ on arrival, were used in these studies. They were initially group-housed (three rats/cage) in $25 \times 45 \times 15 \mathrm{~cm}$ plastic cages, maintained on a 12/12-h light/dark cycle (lights on at 0700 hours), and given access to food and water ad libitum. Rats were allowed to acclimatize for a minimum of 4-7 days before any surgical procedures. For 1 week before behavioral testing, the rats were individually housed and maintained on a restricted diet of $14 \mathrm{~g}$ of food pellets per day, with water freely available. Experiments were conducted during the light portion of the cycle, between 0900 and 1700 hours. All procedures were reviewed and approved by the Institutional Animal Care and Use Committee of the University of Texas Health Science Center at San Antonio, and were consistent with NIH guidelines for the care and use of laboratory animals. All efforts were made to minimize animal pain, discomfort or suffering, and the number of rats used.

\section{Attentional Set-Shifting Protocol}

The method was adapted from Birrell and Brown (2000), as described in Lapiz and Morilak (2006). The testing apparatus was a rectangular wooden arena (inner dimensions, length $\times$ width $\times$ height: $71 \times 40 \times 20 \mathrm{~cm}$ ) painted white on all surfaces. A removable, white plexiglas divider separated one-third of the length of the arena from the rest, forming a start box. This also served as a holding area following each trial, allowing the experimenter to clean the arena and change pots. To begin each trial, a rat was placed in the start box, and given access to the rest of the arena by lifting this divider. A white plexiglas panel divided the opposite third of the arena into two sections. At testing, one digging bowl was placed in each section. This separation enabled the experimenter to quickly remove the rat following an incorrect response, thus preventing it from moving to the other bowl to retrieve the bait. The digging bowls consisted of small terracotta pots (internal rim diameter $7 \mathrm{~cm}$; depth $6 \mathrm{~cm}$ ). Each pot was defined by a pair of cues along two stimulus dimensions, the digging medium with which the pot was filled and an odor (see Table 1). To mark each pot with a distinct odor, two drops ( $10 \mu \mathrm{l} / \mathrm{drop})$ of scented aromatic oil (Frontier Natural Brands, Boulder, CO, USA) was applied to the inner rim at least 5 days before use. Then, $3-5 \mu$ l of the same oil was reapplied on the day before testing. A different pot was used for each combination of digging medium and odor, and only one odor was ever applied to a given pot. The bait, which was placed at 
Table I Behavioral Protocol for Attentional Set-Shift Testing

\begin{tabular}{|c|c|c|c|c|}
\hline \multirow[b]{2}{*}{ Discrimination stage } & \multicolumn{2}{|c|}{ Dimensions } & \multicolumn{2}{|c|}{ Example combinations } \\
\hline & Relevant & Irrelevant & $(+)$ & $(-)$ \\
\hline \multirow[t]{2}{*}{ Compound (CD) } & Odor & Medium & Clove/Raffia & Nutmeg/Metallic filler \\
\hline & & & Clove/Metallic filler & Nutmeg/Raffia \\
\hline \multirow[t]{2}{*}{ Intradimensional shift (ID) } & Odor & Medium & Rosemary/Wood balls & Cinnamon/Plastic beads \\
\hline & & & Rosemary/Plastic beads & Cinnamon/Wood balls \\
\hline \multirow[t]{2}{*}{ Reversal 2 (R2) } & Odor & Medium & Cinnamon/Plastic beads & Rosemary/Wood balls \\
\hline & & & Cinnamon/Wood balls & Rosemary/Plastic beads \\
\hline Extradimensional shift (ED) & Medium & Odor & Velvet/Citronella & Crepe/Thyme \\
\hline
\end{tabular}

Representative example of stimulus pairs and the progression through the stages of the attentional set-shifting protocol. In this example, odor was the initial

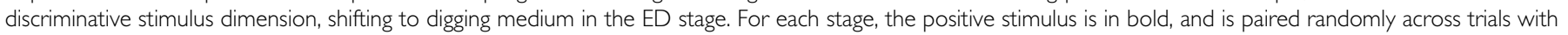
the two stimuli from the irrelevant dimension.

the bottom of the 'positive' pot and buried with the digging medium, was a $1 / 4$ Honey Nut Cheerio (General Mills Cereals, Minneapolis, MN, USA). In all discrimination trials, a small quantity of powdered Cheerio was sprinkled onto the digging medium in the unbaited pot to eliminate the possibility that the rat may locate the bait by smell rather than by learning the discrimination.

Digging was defined as a vigorous displacement of the medium to retrieve the reward buried within the pot. Simply investigating the rim of the pot or the surface of the digging medium with paws or snout without displacing material was not scored as a 'dig', and the trial continued until a 'dig' response was executed. Thus, rats were able to access tactile, visual, and olfactory characteristics of the pots to make their choices based on these stimulus dimensions.

The behavioral procedure entailed 3 days for each rat:

Day 1, habituation: On the first day, the rats were trained to dig reliably in the pots to obtain a food reward. Two unscented pots, both baited, were placed in the home cage for a series of three exposures of $5 \mathrm{~min}$ each. With each exposure, the bait was covered with an increasing amount of sawdust. Once the rat was digging reliably, it was introduced into the test arena, and given three trials to retrieve reward from both the sawdust-filled baited pots.

Day 2, training: The following day, the rats were trained on a series of simple discriminations (SD), to a criterion of six consecutive correct trials. For these trials, the rats first had to learn to associate the food reward with an odor cue (lemon $v s$ rosewood, both pots filled with sawdust). After criterion was reached for the odor discrimination, the pots were changed, and they had to discriminate the digging media (felt strips $v s$ shredded paper, no odor). All rats were trained using the same pairs of stimuli and in the same order. The positive and negative cues for each rat were determined randomly and represented equally. These training stimuli were not used again in later testing trials.

Day 3, testing: Following the training day, the rats were tested on a series of increasingly difficult discriminations (see Table 1). Testing continued at each stage until the rat reached a criterion of six consecutive correct trials, after which testing proceeded to the next stage.

The first stage was a simple discrimination (SD), involving only one stimulus dimension. Half the rats in any group were required at this stage to discriminate between two odors, only one of which was associated with reward, with both pots filled with sawdust. For the other half, this first discrimination involved digging media, with no odors applied to the pots (for the sake of clarity, the remainder of this description will only consider the example beginning with odor discrimination). The second stage was then a compound discrimination (CD), where the second irrelevant stimulus was introduced. Only one odor was associated with reward, as in the SD stage, but two different digging media were now paired randomly with the odors. The third stage was a reversal of this discrimination (R1), in which the same odors and media were used, and odor was still the relevant dimension, but in these trials the negative odor from the previous stage was now positive (ie, associated with the reward), and the positive odor from the previous stage was now negative (no reward). The fourth stage was an intradimensional (ID) shift, wherein odor was still the relevant dimension and the medium was still irrelevant, but all new stimuli were used (ie, new odors and new media). The fifth stage was a reversal of this discrimination (R2), in which the previously negative odor was now positive, as in $\mathrm{R} 1$. The sixth stage required an extradimensional (ED) shift, in which all new stimuli were again introduced, but this time the relevant dimension was also changed, for example, the digging medium became the relevant dimension and 
odor was now irrelevant. Finally, the seventh stage was another reversal (R3), where the previously negative medium was now positive. The assignment of each exemplar in a pair as being positive or negative in a given stage, and the left-right positioning of the pots in the arena on each trial, were randomized in advance. For half the rats, the discriminations began with odor as the relevant cue, as described above, and for half the discrimination began with medium as the salient cue. Table 1 outlines the progression through these stages, and provides examples of the cue combinations used, beginning in this case with an odor discrimination, shifting to medium in the ED shift stage.

The primary dependent measure in this procedure was the number of trials required to reach the criterion of six consecutive correct responses at each test stage (Trials to Criterion, TTC). In addition, we also analyzed the occurrence of set loss errors, a measure adapted from the analysis of errors on the WCST as described by Stuss et al (1983, 2000). In scoring the WCST, with the criterion for successful mastery of a test stage set at 10 consecutive correct responses, Heaton (1981) defined a set loss as an error made after five or more consecutive correct responses, that is, after reaching $50 \%$ or more of criterion. Thus, in the present study, we similarly defined a set loss as an error made after three or more consecutive correct responses, that is, after $50 \%$ or more of the criterion of six consecutive correct responses had been achieved.

Experiment 1: Effects of Acute Systemic Administration of DMI on Extracellular NE Levels in mPFC and on Attentional Set Shift Performance

A total of 15 rats weighing 250-275 g at the time of surgery were anesthetized with a cocktail of ketamine $43 \mathrm{mg} / \mathrm{ml}$, acepromazine $1.4 \mathrm{mg} / \mathrm{ml}$, xylazine $8.6 \mathrm{mg} / \mathrm{ml}, 1.0 \mathrm{ml} / \mathrm{kg}$, i.m., with a $25 \%$ supplement given as needed. Rats were placed in a stereotaxic frame with the incisor bar set at $-3.3 \mathrm{~mm}$, adjusted as needed to achieve a flat skull, indicated by equal DV coordinates for bregma and lambda. A guide cannula (CMA/12; CMA Microdialysis, North Chelmsford, MA), aimed at the $\mathrm{mPFC}$, was implanted using a $10^{\circ}$ lateral approach with the following coordinates relative to bregma: $\mathrm{AP}+2.6 \mathrm{~mm}, \mathrm{ML}+1.4 \mathrm{~mm}$, DV $-1.7 \mathrm{~mm}$, based on the atlas of Paxinos and Watson (1998). Approximately half the rats in each group were implanted on the left side, and half on the right. The guide cannula was anchored to the skull with jewelers screws and dental acrylic, and an obdurator was inserted to maintain patency. During surgery, rats were treated prophylactically with antibiotic (penicillin $G$, $300000 \mathrm{IU} / \mathrm{ml}, 1.0 \mathrm{ml} / \mathrm{kg}$, s.c.), and housed singly until testing.

Beginning 3 days after surgery, food was restricted to $14 \mathrm{~g} /$ day, with water freely available, for 7 days before testing. Rats underwent the habituation and training sessions as described above. On the testing day, rats were transported to the testing room in their home cage, the obdurator was removed, and a microdialysis probe (CMA/ 12), with molecular weight cutoff of $20 \mathrm{kDa}$ and $4 \mathrm{~mm}$ of active membrane, was inserted into the guide cannula. The probe extended $4 \mathrm{~mm}$ beyond the tip of the guide, centering the active membrane within the mPFC. The probe was perfused with artificial cerebrospinal fluid $(147 \mathrm{mM} \mathrm{NaCl}$,
$2.5 \mathrm{mM} \mathrm{KCl}, 1.3 \mathrm{mM} \mathrm{CaCl}_{2}, 0.9 \mathrm{mM} \mathrm{MgCl}_{2}, \mathrm{pH} 7.4$ ) at a flow rate of $1.0 \mu \mathrm{l} / \mathrm{min}$. Animals were allowed a $2 \mathrm{~h}$ equilibration period before sample collection was initiated. Sample collection time was $30 \mathrm{~min}$, resulting in a sample volume of $30 \mu \mathrm{l}$, collected into a tube containing $2.5 \mu \mathrm{l}$ of stabilizing solution $(0.2 \mu \mathrm{M}$ EDTA, $0.2 \mu \mathrm{M}$ ascorbic acid, $0.2 \mathrm{M}$ perchloric acid). Following collection of four baseline samples in the home cage, rats were given a systemic injection of either saline vehicle $(2 \mathrm{ml} / \mathrm{kg}$, i.p.; $n=8)$ or DMI (15 mg/kg; Sigma, St Louis, MO; $n=7)$. In a previous study, we found this dose of DMI to produce plasma levels comparable to those obtained with the chronic drug treatment employed in Experiment 2 (see Garcia et al, 2004). However, because of the time required for behavioral testing after acute drug administration, plasma DMI levels were not obtained in the present study. Immediately after drug injection, rats were placed into the testing arena and behavioral testing commenced. For collection of all microdialysis samples, a 15 min delay was incorporated into the procedure to allow for the dead volume between the active membrane and the sample collection outlet.

From our previous study (Lapiz and Morilak, 2006), it was clear that some of the test stages would require less time for criterion to be reached than the $30 \mathrm{~min}$ microdialysis sample time. Thus, sampling over some of the behavioral tasks was combined: a single sample was collected during $\mathrm{SD}$ and $\mathrm{CD}$ testing (SD/CD), and also during ID and R2 testing (ID/R2). By contrast, the time required to complete the other test stages was sufficient to allow collection of a single sample during each (R1, ED, and R3).

Thus, each sample contained a minimum of $30 \mu \mathrm{l}$ dialysate $+2.5 \mu \mathrm{l}$ stabilizing solution. From this, the amount of NE in a standard volume of $27.5 \mu \mathrm{l}$ was measured for each sample by high-performance liquid chromatography with coulometric detection (Coulochem2, ESA Inc., East Chelmsford, MA). The mobile phase contained $60 \mathrm{mM}$ sodium phosphate, $75 \mu \mathrm{M}$ EDTA, $1.5 \mathrm{mM}$ sodium 1-octanesulfonic acid, $6 \%$ methanol, $\mathrm{pH} 4.6$, at a flow rate of $0.6 \mathrm{ml} / \mathrm{min}$. Under these conditions, NE had a retention time of approximately $7.8 \mathrm{~min}$. The amount of NE in each sample was quantified against a calibration curve run daily, ranging from 0.0 to $25.0 \mathrm{pg} / \mathrm{sample}$, with a detection limit of approximately $0.5 \mathrm{pg} /$ sample.

\section{Experiment 2: Effects of Chronic DMI Treatment on Extracellular NE Levels in mPFC Measured Concurrently with Attentional Set Shift Behavioral Performance}

Initially, 17 rats were used to test the effects of chronic DMI treatment on behavior in the attentional set-shifting task. They were randomly assigned to two groups defined by the drug delivered chronically by osmotic minipump. Rats weighing 220-240 g at the time of surgery were anesthetized with a cocktail of ketamine $43 \mathrm{mg} / \mathrm{ml}$, acepromazine $1.4 \mathrm{mg} /$ $\mathrm{ml}$, xylazine $8.6 \mathrm{mg} / \mathrm{ml}, 1.0 \mathrm{ml} / \mathrm{kg}$, i.m., with a $25 \%$ supplement given as needed. Osmotic minipumps (2ML4, Alzet Corp., Palo Alto, CA), preloaded with either vehicle (10\% ethanol; $n=8)$ or DMI $(n=9)$ at a concentration calculated to deliver $7.5 \mathrm{mg} / \mathrm{kg} /$ day of the free base (approximately $40 \mathrm{mg} / \mathrm{ml}$ ), were implanted intraperitoneally via a ventral midline incision. Following surgery, rats were treated with 
antibiotic (penicillin G, $300000 \mathrm{IU} / \mathrm{ml}, 1.0 \mathrm{ml} / \mathrm{kg}$, s.c.), and housed singly until testing (21 days). Beginning 14 days after surgery, food was restricted to $14 \mathrm{~g}$ /day with water freely available for 7 days before testing on day 21 of drug treatment. Habituation, training, and testing were performed exactly as described above.

A second cohort of 18 rats underwent surgery for both osmotic minipump implantation and stereotaxic implantation of a microdialysis guide cannula. These rats, weighing $220-240 \mathrm{~g}$ at the time of surgery, were anesthetized, and osmotic minipumps loaded with vehicle (10\% ethanol, $n=7)$ or DMI $(7.5 \mathrm{mg} / \mathrm{kg} /$ day; $n=11)$ were implanted intraperitoneally, as above. Rats were then placed in a stereotaxic frame and implanted with a guide cannula aimed at mPFC using a $10^{\circ}$ lateral approach, as in Experiment 1. Approximately half the rats in each group were implanted on the left side, and half on the right.

Beginning 14 days after surgery, food was restricted to $14 \mathrm{~g} /$ day with water freely available for 7 days before testing on day 21 of drug treatment. Habituation and training were conducted as above. On the testing day, in rats implanted with a guide cannula, the obdurator was removed and a microdialysis probe was inserted into the guide, centering the active membrane within mPFC. Animals were allowed a $2 \mathrm{~h}$ equilibration period before sample collection was initiated, also as above. Following collection of four $30 \mathrm{~min}$ baseline samples in the home cage, the rat was placed in the testing arena. As in Experiment 1, a single sample was collected during the combined SD and $\mathrm{CD}$ test stages $(\mathrm{SD} /$ $\mathrm{CD}$ ), and during the combined ID and R2 stages (ID/R2), whereas single samples were collected during each of the other test stages (R1, ED, and R3). The amount of NE in the microdialysate samples was measured by HPLC, as above.

\section{Data Analyses}

In all experiments, the investigator conducting the behavioral test was blind to the drug treatment condition of the rats. Data from the SD training sessions on day 2 were first compared by repeated measures ANOVA to ensure that training was equivalent between the drug treatment groups. For analysis of data collected on the testing day, Trials to Criterion (TTC) were analyzed by two-way ANOVA (Drug $\times$ Stage) with repeated measures over Stage. Where significant main effects or interactions were indicated, post hoc analyses were performed using Newman-Keuls test to identify differences between drug groups at specific test stages. Set loss errors were similarly analyzed. For all analyses, significance was determined at $P<0.05$.

In microdialysis experiments, to first test for any differences in baseline NE levels collected in mPFC of vehicle- and DMI-treated rats, the mean of the four baseline samples was calculated for each animal, and these were compared by Student's $t$-test. As baseline NE levels were significantly elevated in rats treated chronically with DMI, changes in NE levels during performance of the attentional set-shifting behavioral test were then analyzed in two ways. First, to test for changes in the absolute amount of $\mathrm{NE}$ collected in the samples, expressed as $\mathrm{pg} / \mathrm{sample}$, a one-way ANOVA with repeated measures over Time was conducted, split by Drug. In addition, all sample values were converted

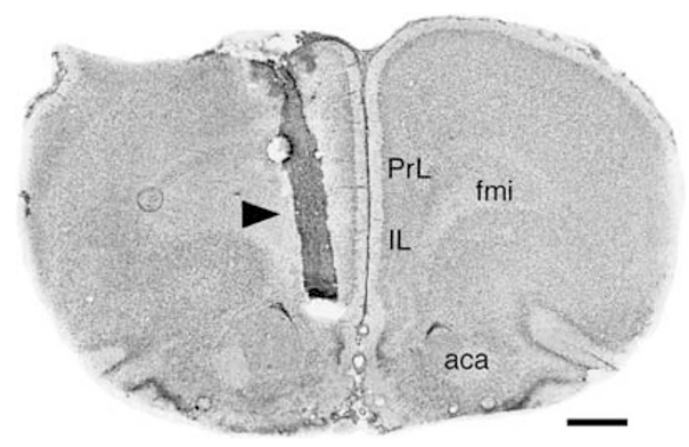

Figure I Representative photomicrograph of a cresyl violet-stained section through mPFC, corresponding to plate 9 in the atlas of Paxinos and Watson (1998), showing the track of a microdialysis guide cannula implanted unilaterally in mPFC. The microdialysis probe extended $4 \mathrm{~mm}$ beyond the tip of the guide cannula, centering the active membrane in mPFC (arrowhead). Abbreviations: aca, anterior commissure, anterior limb; fmi, forceps minor, internal capsule; IL, infralimbic cortex; PrL, prelimbic cortex. Scale bar $=1 \mathrm{~mm}$.

to a percent of the mean baseline value for each rat, and a two-way ANOVA (Drug $\times$ Time), with repeated-measures over Time, was conducted to allow a comparison of the relative magnitude of any change in extracellular NE levels measured in $\mathrm{mPFC}$ during performance of the behavioral test in the two drug groups, after normalizing for baseline differences. Where significant main effects or interactions were indicated using either approach, post hoc analyses were performed using Newman-Keuls test. Significance in all analyses was determined at $P<0.05$.

At the end of each experiment, rats were killed by rapid decapitation, and trunk blood was collected for analysis of plasma DMI levels (assays conducted by Dr Martin Javors, Department of Psychiatry, UTHSCSA). Any DMI-treated subjects who exhibited extreme low or high plasma DMI levels $(<100 \mathrm{ng} / \mathrm{ml}$ or $>1000 \mathrm{ng} / \mathrm{ml})$ were eliminated $a$ priori from subsequent analyses. This resulted in elimination of only one rat for low plasma DMI levels; none exhibited excessively high values. For microdialysis studies, brains were removed for histological determination of probe placement. A representative example of a microdialysis cannula track localized to the MPFC is shown in Figure 1. Any case in which the probe track was located outside the target region in MPFC was likewise eliminated $a$ priori from subsequent analyses. Misplaced probe localization resulted in elimination of two rats. Subjects eliminated for any reason were not included in the reported total number of rats used.

\section{RESULTS}

Experiment 1: Effects of Acute Systemic Administration of DMI on Extracellular NE Levels in MPFC and on Attentional Set Shift Performance

All rats learned to dig in the baited bowls during habituation, and all learned the SD comparably during training, averaging fewer than 10 trials to reach criterion, with no pre-existing differences between the randomly assigned groups $\left(\mathrm{F}_{1,13}=0.81, P=0.38\right)$. Figure 2 shows the effects of acute systemic DMI administration $(15 \mathrm{mg} / \mathrm{kg}$, 


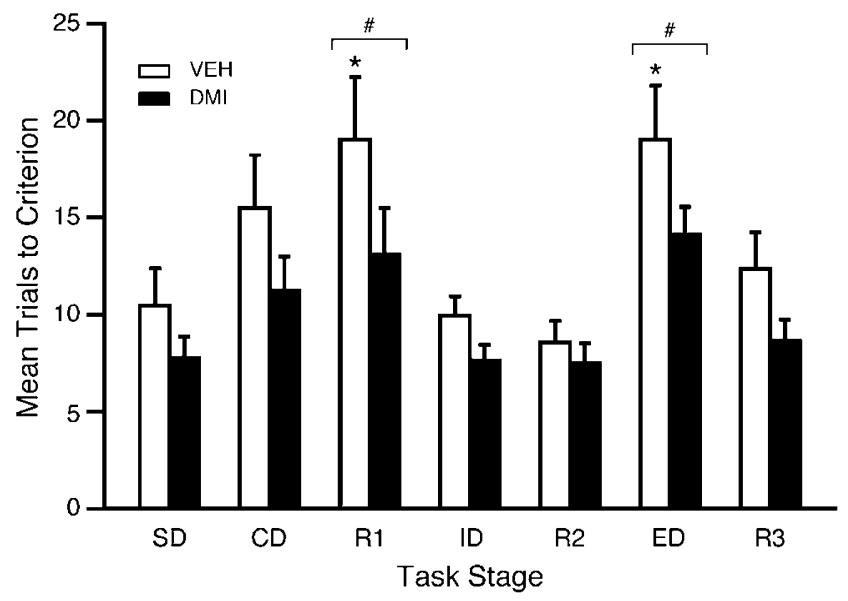

Figure 2 Effects of acute DMl injection ( $15 \mathrm{mg} / \mathrm{kg}$, i.p.) on performance in the attentional set-shifting test, measured by the number of trials to criterion for each test stage (mean $\pm S E M)$. Significantly more trials were required to reach criterion on the $\bar{R} I$ and $E D$ tasks compared to other stages (Main effect of Stage: ${ }^{\#} P<0.05$ compared to other task stages collapsed across drug treatment groups). This effect was seen in the vehicle control group only ${ }^{*} P<0.05$ compared to other task stages within the same drug treatment group). Although acute DMI treatment significantly improved performance overall, there was no significant difference between treatment groups on any individual task stage.

i.p.) on the trials to criterion (TTC) for each stage of the attentional set-shifting test. ANOVA revealed significant main effects of Drug $\left(\mathrm{F}_{1,78}=9.123, P<0.01\right)$ and Stage $\left(\mathrm{F}_{6,78}=7.551, P<0.0001\right)$, but no Drug $\times$ Stage interaction $\left(\mathrm{F}_{6,78}=0.409, P=0.871\right)$. For the main effect of Stage, post hoc comparisons showed that significantly more trials were required to reach criterion during $\mathrm{R} 1$ and $\mathrm{ED}$ than in SD, ID, and R2 (Figure 2), reflecting the relatively greater difficulty of the reversal tasks and the extradimensional setshifting task, as reported previously (Birrell and Brown, 2000; Lapiz and Morilak, 2006). Moreover, within drugtreatment groups, this stage effect was present in the vehicle-treated rats but not in rats treated acutely with DMI, who showed a moderate improvement in performance on the reversal and ED tasks (Figure 2). Nonetheless, although ANOVA indicated that DMI-treated rats performed better overall than the vehicle-treated controls, post hoc comparisons between groups revealed no significant differences in performance on any of the specific task stages.

When set loss errors were similarly analyzed, there were no significant effects of either Stage $\left(\mathrm{F}_{6,78}=1.22, P=0.31\right)$ or Drug, although a trend suggesting that DMI-treated rats made fewer set loss errors approached significance $\left(\mathrm{F}_{1,78}=4.59, P=0.052\right)$.

Figure 3 shows the NE levels collected in microdialysate samples from $\mathrm{mPFC}$ at baseline and during performance on the attentional set-shifting test following acute DMI administration. There were no baseline differences between the two randomly assigned treatment groups before acute injection of DMI or vehicle $(t=-1.24, P=0.24)$. Repeated measures ANOVA revealed a significant main effect of Time $\left(\mathrm{F}_{9,117}=5.13, P<0.001\right)$, but not Drug $\left(\mathrm{F}_{1,117}=0.38\right.$, $P=0.57)$. There was, however, a significant Drug $\times$ Time interaction $\left(\mathrm{F}_{9,117}=3.02, P=0.003\right)$. Post hoc analyses showed that NE levels were significantly higher in DMI-
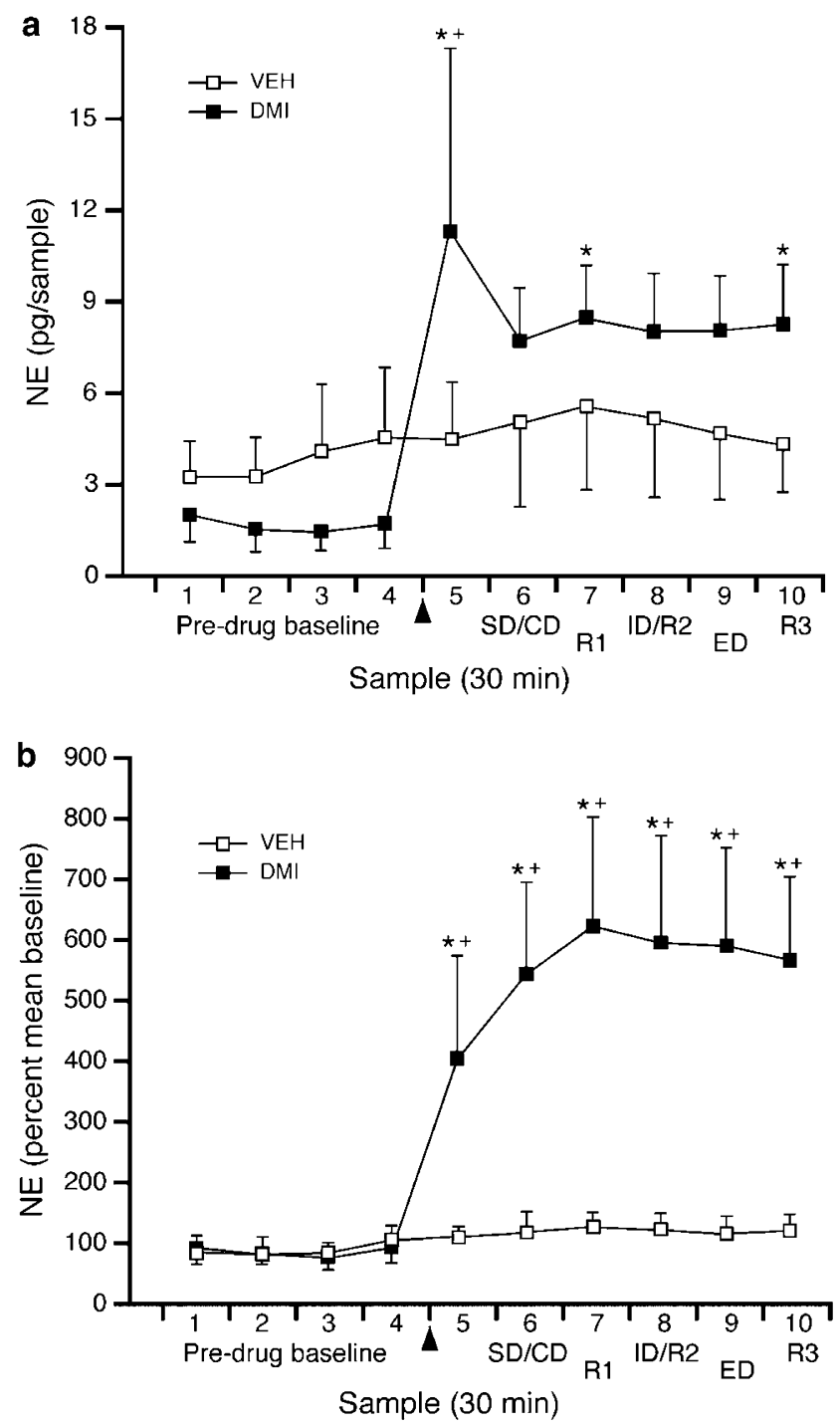

Figure 3 Effects of acute DMI injection ( $15 \mathrm{mg} / \mathrm{kg}$, i.p., arrowhead) on extracellular NE levels in microdialysate samples collected from mPFC. (a) Analysis of absolute NE levels, expressed as pg/sample (mean \pm SEM). NE levels were significantly elevated in MPFC following acute DMI injection. (b) Analysis of data converted to percent of mean baseline. NE levels increased significantly following injection, and the increase was maintained at a stably elevated level during behavior on all stages of the AST. There were no differences between stages during performance of the behavioral test in either drug group. In both panels: $* P<0.05$ compared to baseline samples in the same treatment group; ${ }^{+} P<0.05$ compared to the corresponding time point in the vehicle-injected control group.

treated rats compared to vehicle-treated rats in samples collected after drug injection $(11.47 \pm 5.75 \mathrm{pg} / \mathrm{sample}$ in DMI-treated rats compared to $4.66 \pm 1.66 \mathrm{pg} / \mathrm{sample}$ in controls; $P<0.05)$. The elevation in $\mathrm{NE}$ was maintained for the duration of testing, although post hoc comparisons between groups were significant only for the sample collected immediately following injection and during the R1 and R3 reversal tasks (Figure 3a). Within the treatment groups, there were no significant differences in NE levels collected in mPFC across tasks. When the data were analyzed as percent of mean baseline, ANOVA revealed significant effects of Drug $\left(\mathrm{F}_{1,117}=14.76, P=0.002\right)$ and 
Time $\left(\mathrm{F}_{9,117}=8.14, P<0.001\right)$ and a significant Drug $\times$ Time interaction $\left(\mathrm{F}_{9,117}=6.27, P<0.001\right.$; Figure $\left.3 \mathrm{~b}\right)$. Post hoc analyses showed that acute injection of DMI significantly increased NE in $\mathrm{mPFC}$ compared both to pre-injection baseline and to vehicle-injected controls. The significant increase in NE was maintained for the duration of the behavioral testing period, but NE levels did not vary during performance of the individual tasks in either group (Figure 3b).

\section{Experiment 2: Effects of Chronic DMI Treatment on Extracellular NE Levels in mPFC Measured Concurrently with Attentional Set Shift Behavioral Performance}

Chronic treatment resulted in mean plasma DMI levels of $250.24 \pm 35.59 \mathrm{ng} / \mathrm{ml}$. A comparison of the behavioral data from the subset of rats who were implanted for microdialysis and those who were not revealed no significant difference (for the Microdialysis factor: $\mathrm{F}_{1,31}=1.25$, $P=0.27)$. Thus, the data from these subsets of rats were pooled into otherwise identically treated drug-treatment groups. There were no baseline differences in SD performance of the two drug-treatment groups on the training day $\left(\mathrm{F}_{1,33}=0.85, P=0.77\right)$. Figure 4 shows the effects of chronic (21 days) administration of DMI on the number of trials to criterion for each stage of the attentional set-shifting test. Repeated measures ANOVA revealed significant main effects of Drug $\left(\mathrm{F}_{1,198}=11.44, P=0.002\right)$ and Stage $\left(\mathrm{F}_{6,198}=25.32, P<0.001\right)$, and a significant Drug $\times$ Stage interaction $\left(\mathrm{F}_{6,198}=3.07, P=0.007\right)$. As in Experiment 1 , post hoc analysis of the main effect of Stage across drug

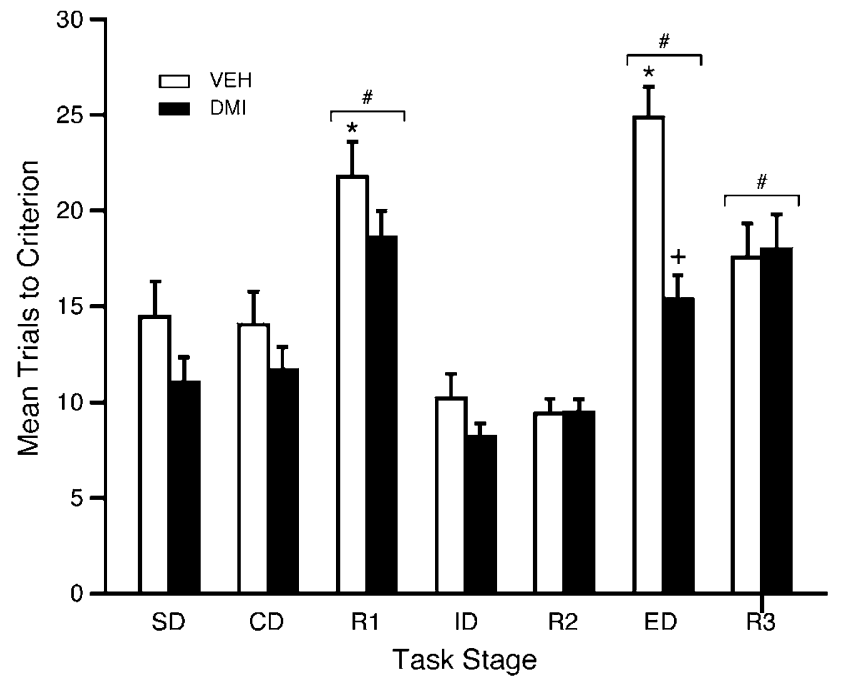

Figure 4 Effects of chronic DMl treatment $(7.5 \mathrm{mg} / \mathrm{kg} /$ day for 21 days $)$ on performance in the attentional set-shifting test, measured by the number of trials to criterion for each test stage (mean \pm SEM). Significantly more trials were required to reach criterion on the RI, ED, and R3 tasks compared to other stages (Main effect of Stage: ${ }^{\#} P<0.05$ compared to other task stages collapsed across drug treatment groups). This effect was seen in the vehicle control group only $(* P<0.05$ compared to other task stages within the same drug treatment group). In addition, however, chronic treatment with DMI significantly improved performance specifically on the ED set shift task $\left({ }^{+} P<0.05\right.$ compared to the same stage in the vehicle-treated control group). groups showed the R1, R3, and ED stages to be significantly more difficult than the other task stages. Further, post hoc comparisons showed that DMI-treated rats performed significantly better than vehicle-treated rats, and that their performance was specifically enhanced on the ED set shift task (Figure 4).

Analysis of set loss errors also revealed a significant effect of Stage $\left(\mathrm{F}_{6,198}=5.57, P<0.001\right)$, but not Drug $\left(\mathrm{F}_{1,198}=0.17\right.$, $P=0.68)$, and a significant Drug $\times$ Stage interaction
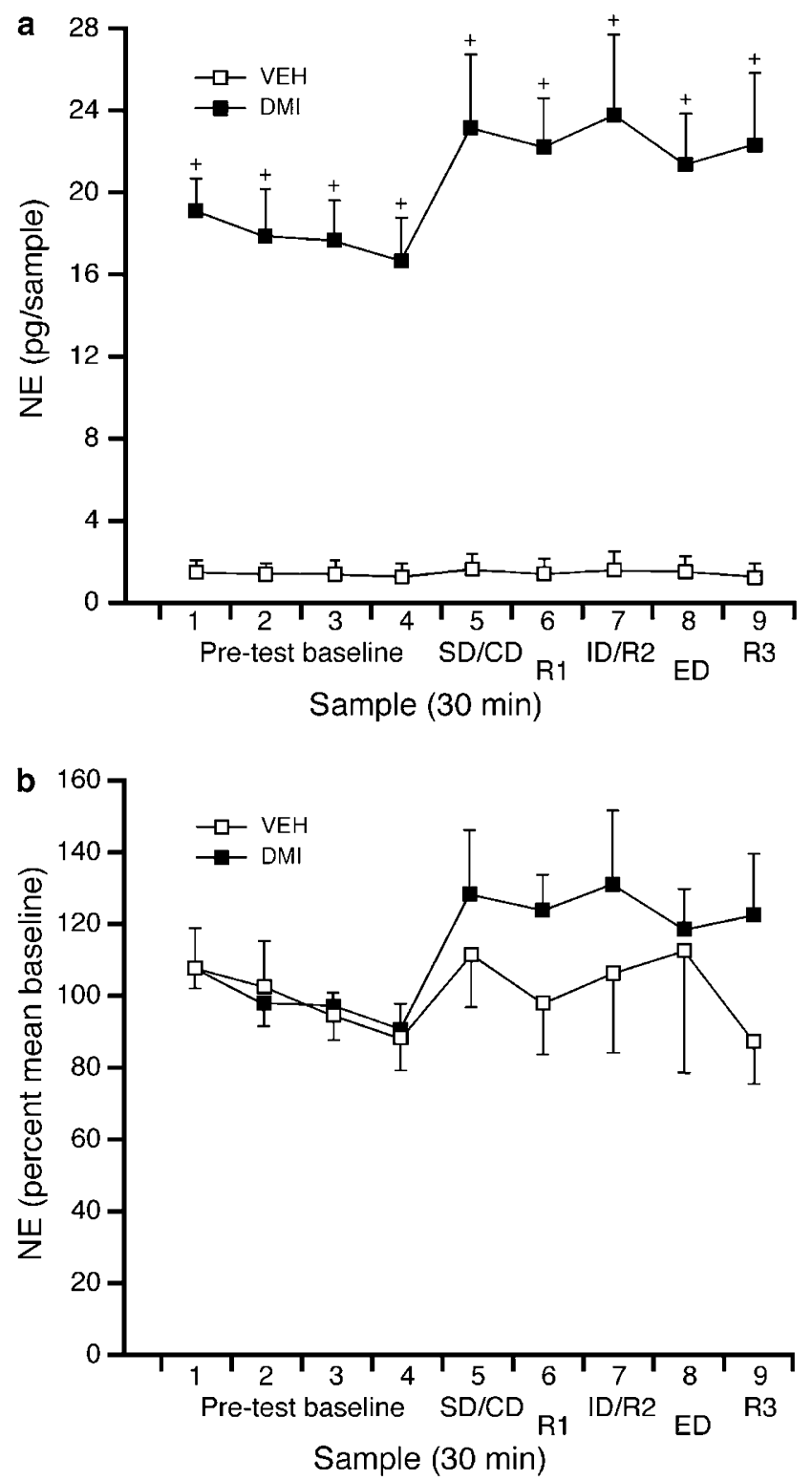

Figure 5 Effects of chronic DMl treatment $(7.5 \mathrm{mg} / \mathrm{kg} /$ day for 21 days $)$ on extracellular NE levels in microdialysate samples collected from mPFC. (a) Analysis of absolute NE levels, expressed as pg/sample (mean \pm SEM). Rats treated chronically with DMI had significantly higher NE levels in all samples collected from mPFC compared to vehicle-treated control rats $\left({ }^{+} P<0.05\right)$. (b) Analysis of data expressed as percent of mean baseline. There was no significant effect over Sample in either drug treatment condition; although there was a trend suggesting an increase in extracellular NE levels during behavioral performance on the attentional set-shifting test, especially in the DMI-treated group, this effect only approached significance $(P=0.052)$. 
$\left(\mathrm{F}_{6,198}=2.39, \quad P<0.05\right)$. Post hoc comparisons showed significantly more set loss errors made during ED compared to $\mathrm{SD}, \mathrm{R} 1, \mathrm{ID}$, and $\mathrm{R} 3$. Moreover, DMI-treated rats made significantly fewer set loss errors than vehicle controls specifically during the ED task.

Figure 5 shows the NE levels measured in microdialysate samples collected from mPFC at baseline and during performance of the attentional set-shifting test in rats treated chronically with vehicle or DMI. Baseline NE levels were significantly elevated in DMI-treated rats $(18.11 \pm 1.57 \mathrm{pg} / \mathrm{sample}$ compared to $1.80 \pm 0.23 \mathrm{pg} / \mathrm{sample}$ in vehicle-treated controls; $t=8.18, P<0.001)$. ANOVA for absolute NE levels (pg/sample, Figure 5a) indicated a significant main effect of Drug $\left(\mathrm{F}_{1,128}=60.99, P<0.001\right)$, but not Time $\left(\mathrm{F}_{8,128}=1.81, P=0.08\right)$, and no significant Drug $\times$ Time interaction $\left(\mathrm{F}_{8,128}=1.61, P=0.13\right)$. Normalization of the data to be expressed as percent of mean baseline allowed a comparison of the magnitude of any potential changes in NE levels in MPFC during behavioral testing in the two groups (Figure 5b). This analysis of course obviated the main effect of Drug $\left(\mathrm{F}_{1,128}=1.37\right.$, $P=0.26$ ), and although NE levels appeared to increase slightly during all stages of behavioral testing relative to pre-testing baseline, especially in the DMI-treated group, the main effect of Time was also not significant $\left(F_{8,128}=1.70, P=0.10\right)$. Consistent with the modest elevation in NE levels seen during overall behavioral performance, a correlation analysis of trials to criterion specifically on the ED set shift stage with NE levels, collected in mPFC of DMI-treated rats used for microdialysis, revealed a similarly modest inverse correlation that also did not achieve significance $(r=-0.34, P=0.31)$.

\section{DISCUSSION}

In this study, we found that NE reuptake blockade by DMI enhanced cognitive performance on an attentional setshifting test, consistent with our previous observation that tonic elevation of NE neurotransmission in mPFC enhanced performance on this test (Lapiz and Morilak, 2006). Both acute and chronic DMI treatment increased extracellular NE levels in the mPFC, measured by in vivo microdialysis, and both treatments improved overall performance on the behavioral test. However, tonic extracellular NE levels were elevated to a greater extent with chronic treatment, and the behavioral effect of DMI was correspondingly more robust after chronic treatment. Only after chronic treatment was the effect sufficient to produce a significant improvement on any individual task stage, and specifically on the ED setshifting task.

The extradimensional set shift places complex demands on neural circuits mediating processes that include attention, short-term memory, and cognitive flexibility. The difficulty of this task is reflected in the consistently higher number of trials required to reach criterion, evident both in this study and in previous studies (Birrell and Brown, 2000; Lapiz and Morilak, 2006; McAlonan and Brown, 2003; Tunbridge et al, 2004). In this task, not only were the rats required to learn new discriminations using new sets of stimuli but they were also required to switch their learning strategy, shifting attention away from the previously salient stimulus dimension and attending to the previously irrelevant dimension (Chen et al, 2004; Lapiz and Morilak, 2006). Lesions of the dorsolateral PFC in primates, and of the analogous $\mathrm{mPFC}$ in rats, specifically impaired performance on ED set-shifting tasks (Birrell and Brown, 2000; Dias et al, 1996). Similar impairment of performance on the WCST was seen in patients with lesions of the dorsolateral prefrontal cortex (Stuss et al, 2000), and in patients suffering from a variety of neuropsychiatric disorders in which mPFC dysfunction has been implicated (Fossati et al, 1999; Goldstein et al, 2002; Moritz et al, 2002). These results indicate that optimal functioning of the mPFC is important in the cognitive processes that underlie attentional setshifting. Moreover, elevated NA neurotransmission has been shown to facilitate a number of cognitive processes related to $\mathrm{mPFC}$ functioning, including vigilance, selective attention, learning, and memory (Aston-Jones et al, 1991, 1999; Dalley et al, 2004; Devauges and Sara, 1991; Dias et al, 1996). We have shown previously that elevating NA neurotransmission by systemic administration of atipamezole, an $\alpha_{2}$-autoreceptor antagonist, improved performance on the ED set-shifting task, and this improvement was blocked specifically by local microinjection of a postsynaptic $\alpha_{1}$-adrenergic receptor antagonist directly into mPFC (Lapiz and Morilak, 2006).

Electrophysiological studies in primates have shown that the mode of activity in the coeruleo-cortical NA system is closely related to performance in a selective attention task (Aston-Jones et al, 1994, 1999, 2000). In these studies, phasic activation of neurons in the locus coeruleus (LC) was associated with accurate detection and responding to salient, reward-predicting stimuli and not to distractors. However, there was an inverted U-shaped association between tonic baseline firing of LC neurons, related to the state of arousal, and both the phasic excitation of these cells by the salient stimuli and the accuracy of performance in the detection task. At low levels of tonic NA activity, corresponding to low arousal, performance was also low. At moderate levels of baseline NA activity and arousal, phasic activation of NA neurons was greater, and the ability to attend to reward-signaling stimuli and to filter distracting stimuli was optimal, consistent with a state of 'selective attention' and a mode of responding that is most appropriate to maintaining previously established contingencies. At higher levels of tonic NA activity, response accuracy was reduced, and phasic activation of NA neurons by target stimuli was masked by the higher levels of tonic activity (Aston-Jones et al, 1994, 1999, 2000). This corresponds to a state of 'scanning attention' and an increase in vigilance, wherein the animal was more attentive to environmental cues rather than being focused on previously learned contingencies, thereby enabling the potential salience of new cues to be established (AstonJones et al, 1994, 1999, 2000). Thus, the improvement in ED set-shifting capability after chronic DMI treatment may reflect this switch into a mode of 'scanning attention', or enhanced behavioral flexibility, allowing for extinction of a previously established but no longer valid contingency, and the efficient acquisition of new contingencies. Further, this state of enhanced cognitive flexibility may correspond specifically to a state of elevated tonic activity, but reduced phasic excitability of the NA system. 
Chronic treatment with an NE reuptake inhibitor may produce exactly such a change in neurotransmission. Clearance of neurotransmitter from the synapse by presynaptic transporters is the primary mechanism by which the postsynaptic effects of neurotransmitter release are terminated (Giros and Caron, 1993; see Mason et al, 2005; Miller et al, 1999). This allows any subsequent phasic activity in the presynaptic cell to be transmitted faithfully and with temporal fidelity to the postsynaptic neuron by the subsequent release of more neurotransmitter. However, with chronic reuptake blockade, neurotransmitter is not cleared from the synapse and extracellular levels increase to a stably elevated level that is likely to be, to a large extent, disconnected from acute fluctuations in presynaptic activity. Moreover, the greatly elevated extracellular levels of NE obtained with chronic reuptake blockade will also act on presynaptic terminal autoreceptors and somatodendritic autoreceptors to inhibit acute excitability of the presynaptic neuron (Béique et al, 2000; Szabo and Blier, 2001; Szabo et al, 2000; Valentino et al, 1990), further disconnecting neurotransmission from phasic presynaptic activity. Unlike the desensitization of 5-HT1A autoreceptors that has been observed in serotonergic neurons with chronic SSRI treatment, we have found that $\alpha_{2}$-adrenergic autoreceptors remain functional and continue to inhibit NE release after chronic treatment with DMI (Garcia et al, 2004).

In the present study, performance of the attentional setshifting task in both drug- and vehicle-treated control rats was accompanied by a modest, albeit consistent increase in $\mathrm{NE}$ levels in $\mathrm{mPFC}$ relative to baseline. This modest increase in NE transmission, about $15 \%$ above baseline in controls and $30 \%$ in DMI-treated rats (relative to their already greatly elevated baseline), was only marginally significant, and did not appear to be specific to any one behavioral task. This is perhaps more reflective of a general increase in overall arousal than any task-specific processing. The modest increase in $\mathrm{NE}$ is in contrast to a more robust increase seen recently in dopamine levels in mPFC (about $100 \%$ above baseline) during both rule-learning and setshifting tasks (Stefani and Moghaddam, 2006). It was suggested in that paper that the increase in dopamine may have been attributable to a possible shift in the firing pattern of dopaminergic neurons from a tonic to a bursting mode of activity (see Gonon, 1988; Grace, 1991). Such an interpretation may be consistent with the suggestion above, that the enhancement of cognitive flexibility by $\mathrm{NE}$ is related more to a steady elevation in tonic NA neurotransmission, perhaps contributing to an enhanced state of arousal or vigilance, rather than to an increase in phasic NA reactivity or excitability, which may be more effective in facilitating responses evoked specifically by an acute stressor.

It has been increasingly recognized that, in addition to mood symptoms, cognitive dysfunction is a major component of depression and anxiety disorders, and may in fact underlie some of the affective symptoms (Beck, 1976; Beck et al, 1987; Coles and Heimberg, 2002; Mathews and Mackintosh, 1998). Patients with anxiety and depression exhibit cognitive and memory biases for emotionally relevant material, particularly with respect to 'controllability' of stressful life events, a concept long theorized to be central to the development of depression (Seligman, 1974).
It has been suggested that a perseverative attention to perceived threats, themes of loss, personal deficiency, worthlessness, or hopelessness may contribute to a vulnerability to develop affective disorders (Coles and Heimberg, 2002; MacLeod and Hagan, 1992). Consistent with the idea of altered cognitive processes in depression is the growing evidence demonstrating frontal lobe dysfunction in depression. Neuropsychological studies of depressed patients have revealed impairments on executive tests considered to reflect frontal lobe function, for example, verbal fluency tasks, cognitive flexibility, initiation ability, and perseverative errors (see Fossati et al, 1999). Depressed patients show abnormal responses to performance feedback, reduced motivation, diminished cognitive capacity and processing resources, narrowing of attentional focus to depressionrelevant thoughts, and difficulty shifting attention from one affective category to another (see Murphy et al, 1999, 2003). In addition, functional neuroimaging studies have shown associations between cognitive dysfunction and altered activation of the prefrontal cortex in depression (see Rogers et al, 2004). Thus, the present results suggest that the facilitatory effects of NE on cognitive flexibility in the mPFC may contribute to the mechanisms whereby therapeutic drugs that influence NA neurotransmission can exert beneficial cognitive effects in the treatment of depression.

Selective NRIs, including DMI, are effective antidepressants, and the importance of $\mathrm{NE}$ in the mechanism of antidepressant efficacy of these drugs has been demonstrated clinically. Acute depletion of catecholamines by administration of $\alpha$-methylparatyrosine, a selective inhibitor of tyrosine hydroxylase, transiently reversed the antidepressant response in patients treated with DMI, mazindol, or mirtazapine (Delgado et al, 1983, 2002; Miller et $a l, 1996)$. Indeed, there is a general consensus that typical ADs act primarily to facilitate monoaminergic neurotransmission. However, the clinically therapeutic actions of these drugs cannot be attributed solely to their ability to elevate monoamine levels by acute pharmacological blockade of the relevant transporter. The beneficial effects of ADs are only evident after continued chronic administration, the socalled 'therapeutic lag' (Gelenberg and Chesen, 2000). Although their pharmacological effects on transporter function are immediate (Nestler, 1998; Ressler and Nemeroff, 1999), detectable improvements in either global symptomatology or in specific symptoms becomes evident after at least 7-14 days of treatment, whereas significant clinical improvement occurs only after several weeks of treatment, and more complete recovery can take many months (Blier and de Montigny, 1994; Frazer, 1994; Katz et al, 1996/1997, 2004).

Hence, any model addressing potential mechanisms of antidepressant action should reflect an aspect of timedependency. The demonstration of a degree of timedependency in the present study highlights the possible use of the attentional set-shifting paradigm as a model to further investigate the neural mechanisms by which changes in NE neurotransmission and cognition may be involved in the therapeutic actions of ADs. The current results suggest that regulatory alterations in NA neurotransmission induced by chronic treatment with a selective NRI may produce more effective facilitation of tonic NA activity than that produced by acute pharmacological reuptake blockade 
alone. In a broader sense, understanding the mechanisms by which chronic treatment with NRIs and other classes of antidepressants can regulate monoaminergic function to produce their clinical effects may contribute to a better understanding of the etiology of depression, and perhaps to the future development of more effective, rapid, or selective treatment strategies.

\section{ACKNOWLEDGEMENTS}

Expert technical assistance was provided by Ms Selinda Salazar. Support was provided by research grants from the National Institutes of Health (MH53851 and MH72672 to DAM) and the National Alliance for Research on Schizophrenia and Depression (to MDSL). The authors have no conflicts of interest to report, nor any involvement to disclose, financial or otherwise, that may bias the conduct, interpretation, or presentation of this work.

\section{REFERENCES}

Anisman H, Matheson K (2005). Stress, depression, and anhedonia: caveats concerning animal models. Neurosci Biobehav Revs 29: $525-546$.

Aston-Jones G, Chiang C, Alexinsky T (1991). Discharge of noradrenergic locus coeruleus neurons in behaving rats and monkeys suggests a role in vigilance. Prog Brain Res 88: 501-520.

Aston-Jones G, Rajkowski J, Cohen J (1999). Role of locus coeruleus in attention and behavioral flexibility. Biol Psychiatry 46: $1309-1320$.

Aston-Jones G, Rajkowski J, Cohen J (2000). Locus coeruleus and regulation of behavioral flexibility and attention. Prog Brain Res 126: $165-182$.

Aston-Jones G, Rajkowski J, Kubiak P, Alexinsky T (1994). Locus coeruleus neurons in monkey are selectively activated by attended cues in a vigilance task. J Neurosci 14: 4467-4480.

Beck AT (1976). Cognitive Therapy and the Emotional Disorders. Int. University Press: New York.

Beck AT, Brown G, Steer RA, Eidelson JI, Riskind JH (1987). Differentiating anxiety and depression: a test of the cognitive content-specificity hypothesis. J Abnormal Psychol 96: 179-183.

Béïque J-C, de Montigny C, Blier P, Debonnel G (2000). Effects of sustained administration of the serotonin and norepinephrine reuptake inhibitor venlafaxine: I. In vivo electrophysiological studies in the rat. Neuropharmacology 39: 1800-1812.

Birrell JM, Brown VJ (2000). Medial frontal cortex mediates perceptual attentional set shifting in the rat. J Neurosci 20: 4320-4324.

Blier P, de Montigny C (1994). Current advances and trends in the treatment of depression. Trends Pharmacol Sci 15: 220-226.

Chen KC, Baxter MG, Rodefer JS (2004). Central blockade of muscarinic cholinergic receptors disrupts affective and attentional set-shifting. Eur J Neurosci 20: 1081-1088.

Coles ME, Heimberg RG (2002). Memory biases in the anxiety disorders: current status. Clin Psychol Rev 22: 587-627.

Dalley JW, Cardinal RN, Robbins TW (2004). Prefrontal cognitive and executive functions in rodents: neural and neurochemical substrates. Neurosci Biobehav Revs 28: 771-784.

Delgado PL, Moreno FA, Onate L, Gelenberg AJ (2002). Sequential catecholamine and serotonin depletion in mirtazapine-treated depressed patients. Int J Neuropsychopharmacol 5: 63-66.

Delgado PL, Miller HL, Salomon RM, Licinio J, Heninger GR, Gelenberg AJ et al (1983). Monoamines and the mechanism of antidepressant action: effects of catecholamine depletion on mood of patients treated with antidepressant. Pscyhopharmacol Bull 29: 389-396.

Delgado PL, Moreno FA (2000). Role of norepinephrine in depression. J Clin Psychiatry 61: 5-12.

Devauges V, Sara SJ (1991). Memory retrieval enhancement by locus coeruleus stimulation: evidence for mediation by $\beta$ receptors. Behav Brain Res 43: 93-97.

Dias R, Robbins TW, Roberts AC (1996). Primate analogue of the Wisconsin Card Sorting Test: effects of excitotoxic lesions of the prefrontal cortex of the marmoset. Behav Neurosci 110: 872-886.

Dias R, Robbins TW, Roberts AC (1997). Dissociable forms of inhibitory control within prefrontal cortex with an analog of the Wisconsin Card Sort Test: restriction to novel situations and independence from 'on-line' processing. J Neurosci 17: 9285-9297.

Eichenbaum HB, Ross R, Raji A, McGaughy JA (2003). Noradrenergic, but not cholinergic, deafferentation of the infralimbic/ prelimbic cortex impairs attentional set-shifting. Soc Neurosci Abstr 29 Online: Program no. 940.7.

Fossati P, Amar G, Raoux N, Ergis AM, Allilaire JF (1999). Executive functioning and verbal memory in young patients with unipolar depression and schizophrenia. Psychiatry Res 89: 171-187.

Frazer A (1994). Antidepressant drugs. Depression 2: 1-19.

Frazer A (2000). Norepinephrine involvement in antidepressant action. J Clin Psychiatry 61(Suppl 10): 25-30.

Frazer A (2001). Serotonergic and noradrenergic reuptake inhibitors: prediction of clinical effects from in vitro potencies. J Clin Psychiatry 62(Suppl 12): 16-23.

Garcia AS, Barrera G, Burke TF, Ma S, Hensler JG, Morilak DA (2004). Autoreceptor-mediated inhibition of norepinephrine release in rat medial prefrontal cortex is maintained after chronic desipramine treatment. J Neurochem 91: 683-693.

Gelenberg AJ, Chesen CL (2000). How fast are antidepressants? J Clin Psychiatry 61: 712-721.

Giros B, Caron MG (1993). Molecular characterization of the dopamine transporter. Trends Pharmacol Sci 14: 43-49.

Goldapple K, Segal Z, Garson C, Lau M, Bieling P, Kennedy S et al (2004). Modulation of cortico-limbic pathways in major depression: treatment-specific effects of cognitive behavior therapy. Arch Gen Psychiatry 61: 34-41.

Goldstein G, Minshew NJ, Allen DN, Seaton BE (2002). Highfunctioning autism and schizophrenia: a comparison of an early and late onset neurodevelopmental disorder. Arch Clin Neuropsychol 17: 461-475.

Gonon FG (1988). Nonlinear relationship between impulse flow and dopamine released by rat midbrain dopaminergic neurons as studied by in vivo electrochemistry. Neuroscience 24: 19-28.

Gorman JM, Sullivan G (2000). Noradrenergic approaches to antidepressant therapy. J Clin Psychiatry 61(Suppl 1): 13-16.

Grace AA (1991). Phasic versus tonic dopamine release and the modulation of dopamine system responsivity: a hypothesis for the etiology of schizophrenia. Neuroscience 41: 1-24.

Heaton RK (1981). Wisconsin Card Sorting Test Manual. Psychological Assessment Resources: Odesa, FL.

Katz MM, Koslow SH, Frazer A (1996/1997). Onset of antidepressant activity: reexamining the structure of depression and multiple actions of drugs. Depress Anxiety 4: 257-267.

Katz MM, Tekell JL, Bowden CL, Brannan S, Houston JP, Berman $\mathrm{N}$ et al (2004). Onset and early behavioral effects of pharmacologically different antidepressants and placebo in depression. Neuropsychopharmacology 29: 566-579.

Kennedy SH, Evans KR, Kruger S, Mayberg HS, Meyer JH, McCann $S$ et al (2001). Changes in regional brain glucose metabolism measured with positron emission tomography after paroxetine treatment of major depression. Am J Psychiatry 158: 899-905.

Lapiz MDS, Morilak DA (2005). Noradrenergic modulation of cognitive function in rat medial prefrontal cortex as measured 
by attentional set shifting capability. Soc Neurosci Abstr 31 Online: Program no. 644.13.

Lapiz MDS, Morilak DA (2006). Noradrenergic modulation of cognitive function in rat medial prefrontal cortex as measured by attentional set shifting capability. Neuroscience 137: 1039-1049.

MacLeod C, Hagan R (1992). Individual differences in the selective processing of threatening information, and emotional responses to a stressful life event. Behav Res Ther 30: 151-161.

Mason JN, Farmer H, Tomlinson ID, Schwartz JW, Savchenko V, DeFelice LJ et al (2005). Novel fluorescence-based approaches for the study of biogenic amine transporter localization, activity, and regulation. J Neurosci Methods 143: 3-25.

Mathews A, Mackintosh B (1998). A cognitive model of selective processing in anxiety. Cognitive Ther Res 22: 539-560.

McAlonan K, Brown VJ (2003). Orbital prefrontal cortex mediates reversal learning and not attentional set shifting in the rat. Behav Brain Res 146: 97-103.

Miller GW, Gainetdinov RR, Levey AI, Caron MG (1999). Dopamine transporters and neuronal injury. Trends Pharmacol Sci 20: 424-429.

Miller HL, Delgado PL, Salomon RM, Heninger GR, Charney DS (1996). Effects of alpha-methyl-para-tyrosine (AMPT) in drugfree depressed patients. Neuropsychopharmacology 14: 151-157.

Morgan MA, Schulkin J, LeDoux JE (2003). Ventral medial prefrontal cortex and emotional perseveration: the memory for prior extinction training. Behav Brain Res 146: 121-130.

Morilak DA, Barrera G, Echevarria DJ, Garcia AS, Hernandez A, $\mathrm{Ma} S$ et al (2005). Role of brain norepinephrine in the behavioral response to stress. Prog Neuropsychopharmacol Biol Psychiatr 29: $1214-1224$.

Morilak DA, Frazer A (2004). Antidepressants and brain monoaminergic systems: a dimensional approach to understanding their behavioral effects in depression and anxiety disorders. Int $J$ Neuropsychopharmacol 7: 193-218.

Moritz S, Birkner C, Kloss M, Jahn H, Hand I, Haasen C et al (2002). Executive functioning in obsessive-compulsive disorder, unipolar depression, and schizophrenia. Arch Clin Neuropsychol 17: 477-483.

Murphy FC, Michael A, Robbins TW, Sahakian BJ (2003). Neuropsychological impairments in patients with major depressive disorder: the effects of feedback on task performance. Psychol Med 33: 455-467.

Murphy FC, Sahakian BJ, Rubinsztein JS, Michael A, Rogers RD, Robbins TW et al (1999). Emotional bias and inhibitory control processes in mania and depression. Psychol Med 29: 1307-1321.

Nestler EJ (1998). Antidepressant treatments in the 21st century. Biol Psychiatry 44: 526-533.

Owen AM, Roberts AC, Polkey CE, Sahakian BJ, Robbins TW (1991). Extra-dimensional versus intra-dimensional set shifting performance following frontal lobe excisions, temporal lobe excisions or amygdalohippocampectomy. Neuropsychologia 29: 993-1006.

Paxinos G, Watson C (1998). The Rat Brain in Stereotaxic Coordinates, 4th edn. Academic Press: San Diego.

Petre CO, Lapiz MDS, Morilak DA (2005). Effects of acute desipramine treatment on norepinephrine levels in $\mathrm{mPFC}$ and performance of rats on an attentional set-shifting task. Soc Neurosci Abstr 31 Online: Program no. 644.14.

Prasko J, Hornacek J, Zalesky R, Kopecek M, Novak T, Paskova B et al (2004). The change of regional brain metabolism (18FDG PET) in panic disorder during the treatment with cognitive behavioral therapy or antidepressants. Neuro Endocrinol Lett 25 : 340-348.

Ragozzino ME, Detrick S, Kesner RP (1999). Involvement of the prelimbic-infralimbic areas of the rodent prefrontal cortex in behavioral flexibility for place and response learning. J Neurosci 19: 4585-4594.

Ressler KJ, Nemeroff CB (1999). Role of norepinephrine in the pathophysiology and treatment of mood disorders. Biol Psychiatry 46: 1219-1233.

Roberts AC, Robbins TW, Everitt BJ, Muir JL (1992). A specific form of cognitive rigidity following excitotoxic lesions of the basal forebrain in marmosets. Neuroscience 47: 251-264.

Rogers MA, Kasai K, Koji M, Fukuda R, Iwanami A, Nakagome K et al (2004). Executive and prefrontal dysfunction in unipolar depression: a review of neuropsychological and imaging evidence. Neurosci Res 50: 1-11.

Seligman MEP (1974). Depression and learned helplessness. In: Friedman RJ, Katz MM (eds). The Psychology of Depression: Contemporary Theory and Research. Winston-Wiley: New York. pp 83-113.

Sheline YI (2003). Neuroimaging studies of mood disorder effects on the brain. Biol Psychiatry 54: 338-352.

Stefani MR, Moghaddam B (2006). Rule learning and reward contingency are associated with dissociable patterns of dopamine activation in the rat prefrontal cortex, nucleus accumbens, and dorsal striatum. J Neurosci 26: 8810-8818.

Stuss DT, Benson DF, Kaplan EF, Weir WS, Naeser M, Lieberman I et al (1983). The involvement of orbitofrontal cerebrum in cognitive tasks. Neuropsychologia 21: 235-248.

Stuss DT, Levine B, Alexander MP, Hong J, Palumbo C, Hamer L et al (2000). Wisconsin Card Sorting Test performance in patients with focal frontal and posterior brain damage: effects of lesion location and test structure on separable cognitive processes. Neuropsychologia 38: 388-402.

Szabo ST, Blier P (2001). Effect of the selective noradrenergic reuptake inhibitor reboxetine on the firing activity of noradrenaline and serotonin neurons. Eur J Neurosci 13: 2077-2087.

Szabo ST, de Montigny C, Blier P (2000). Progressive attenuation of the firing activity of locus coeruleus noradrenergic neurons by sustained administration of selective serotonin reuptake inhibitors. Int J Neuropsychopharmacol 3: 1-11.

Tollefson GD (1996). Cognitive function in schizophrenic patients. J Clin Psychiatry 57: 31-39.

Tunbridge EM, Bannerman DM, Sharp T, Harrison PJ (2004). Catechol-O-methyltransferase inhibition improves set-shifting performance and elevates stimulated dopamine release in the rat prefrontal cortex. J Neurosci 24: 5331-5335.

Valentino RJ, Curtis AL, Parris DG, Wehby RG (1990). Antidepressant actions on brain noradrenergic neurons. J Pharmacol Exper Ther 253: 833-840. 